\title{
Escala de Actitudes hacia el Endeudamiento: valide $z$ factorial y perfiles actitudinales en estudiantes universitarios chilenos"
}

\author{
Scale Attitude toward Indebtedness: factorial validity and \\ attitudinal profiles in Chilean university students
}

Recibido: enero 25 de 2011 | Revisado: mayo 14 de 2011 | Aceptado: junio 4 de 2011

\author{
MARIANELA DENEGRI CORIA ** \\ Daniel CaBezas GaEte \\ CARLOS DEL VALLE RojaS \\ YÉSSICA GONZÁLEZ GÓMEZ \\ Jocelyne SePÚlvedA ARAVENA \\ Universidad de la Frontera, Temuco, Chile.
}

\section{RESUMEN}

El número de estudiantes universitarios que presentan un endeudamiento temprano se ha incrementado en los últimos años, lo que representa un riesgo potencial para su estabilidad financiera. La presente investigación analiza la validez factorial de la Escala de Actitudes hacia el Endeudamiento (Denegri et al., 1999) y determina la existencia de tipología de perfiles actitudinales hacia el endeudamiento, en una muestra de 984 estudiantes universitarios chilenos con edades entre 18 y 25 años. Los resultados señalan la presencia de dos factores independientes denominados Hedonismo y Austeridad, a partir de los cuales se determinaron cuatro perfiles de actitudes hacia el endeudamiento llamados: austero, difuso, hedonista y ambivalente, que podrían estar a la base de comportamientos de aceptación o rechazo del endeudamiento.

Para citar este artículo. Denegri, M., Cabezas, D. del Valle, C., González, Y. \& Sepúlveda, J. (2012). Escala de Actitudes hacia el Endeudamiento: validez factorial y perfiles actitudinales en estudiantes universitarios chilenos. Universitas Psychologica, 11(2), 497-509.

Esta investigación original forma parte del Proyecto No 1090179, titulado Alfabetización Económica y patrones de consumo y endeudamiento en estudiantes de pedagogía: hacia un modelo explicativo, financiado por el Fondo Nacional de Ciencia y Tecnología (FONDECYT) de la Comisión Nacional de Ciencia y Tecnología de Chile.

** Dirigir correspondencia al E-mail: mdenegri@ ufro.cl. Departamento de Psicología. Universidad de La Frontera, Casilla 54-D. Temuco, Chile. Emails de los demás autores: daniel.cabezas@gmail. com,delvalle@ufro.cl,jgonza@ufro.cl, jocelynesepulveda@yahoo.es. ResearcherID: Denegri, M. F-3320-2012; Cabezas, D. F-3300-2012; del Valle, C. F-3341-2012; Sepúlveda, J. F-3340-2012.

Palabras clave autores

Actitudes, endeudamiento, perfiles actitudinales.

Palabras clave descriptores

Validez, Escala de Actitudes hacia el Endeudamiento, psicometría.

\section{A B S T R A C T}

The number of university students who present an early indebtedness has increased in the last years, which represents a potential risk for his financial stability. This study examined validity factorial of the Scale of Attitudes toward the Indebtedness (Denegri et al., 1999) and determines the existence of profile attitudinal typology towards the indebtedness in a sample of 984 university Chilean students with ages between 18 and 25 years. The results indicate the presence of two independent factors named Hedonism and Austerity, from which four profiles of attitudes decided towards the indebtedness named: austere, diffuse, hedonistic and ambivalent those who might be to the base of his behaviors of acceptance or rejection of the indebtedness. Key words authors

Attitudes, indebtedness, attitudes profile.

Key words plus

Validity, Scale of Attitudes towards the Indebtedness, psychometry. 


\section{Introducción}

La sociedad contemporánea ha sido descrita como una cultura de sobreconsumo y de endeudamiento (Chien \& DeVaney, 2001), caracterizada por una mayor facilidad para el acceso al crédito y como resultado de ello la gente se ha vuelto más tolerante y aceptadora del endeudamiento, como una forma de acceso habitual a aquellos bienes y servicios deseados (Denegri, 2007). De esta forma, el crédito y el endeudamiento se convierten en elementos constitutivos de los procesos de socialización económica familiar y social, presentándose en segmentos de consumidores cada vez más jóvenes (Amar, Abe1lo, Denegri \& Llanos, 2007; Denegri, Palavecinos, Gempp \& Caprile, 2005; Descouvières, 1998).

Según Luna (1998), entre las variables que explicarían esta situación destacan el apoyo social a la deuda, la comparación social, el tipo de locus de control y las actitudes y estilos de manejo del dinero. Desde la perspectiva de la comparación social, los individuos consumen de acuerdo con sus percepciones de la norma para su grupo de referencia. Si la comparación social es con grupos que están sobre el estatus socioeconómico real, esta puede ser una variable subyacente que lleve a los individuos a gastar incluso más allá de sus recursos monetarios, para adquirir productos que ellos consideran propios del grupo de su aspiración. En consecuencia, si tienen altos ingresos en relación con su grupo de referencia, tenderán a ahorrar el excedente; por el contrario, si cuentan con unos ingresos relativamente bajos en relación con su grupo de referencia, se mostrarán más dispuestos a contraer deudas (Wärneryd, 1999). Esto acompañado de la facilidad para optar al crédito y las actitudes sociales de aceptación del endeudamiento han creado un ambiente en el que muchos consumidores creen que es imposible sobrevivir si no se adquieren deudas (Avard, Manton, English \& Walker, 2005; Lea, Webley \& Levine, 1993).

Otros factores analizados han sido la tendencia a valorar más la situación actual que la futura, incluso si racionalmente la situación futura es más ventajosa, y el efecto del locus de control, donde se observa que los sujetos con locus externo tenderían más a endeudarse que aquellos con uno interno (Lea et al., 1993; Lea, Webley \& Walker, 1995; Livingstone \& Lunt, 1992; Watson, 2003).

En cuanto a la influencia de las actitudes, la literatura provee evidencia de que los individuos que tienen actitudes más positivas hacia el crédito tienden a usar con más frecuencia el endeudamiento, como una forma de satisfacer sus necesidades de consumo (Chien \& DeVaney, 2001; Godwin, 1997; Kim \& DeVaney, 2001). Durkin (2000) sugiere que el desarrollo de estas actitudes está influenciado por las experiencias de consumo, tanto directas como vicarias, y no solo por la experiencia concreta del individuo. Por esto, las actitudes personales hacia el crédito han cambiado dramáticamente en la última década, en la medida en que la propia sociedad de consumo acepta que el endeudamiento es un componente clave propio del estilo de vida financiero de los consumidores actuales (Zuckerman, 2000).

Winerman (2004) encuentra que las actitudes correlacionan más fuertemente que otros factores psicológicos, y que aquellas personas con actitudes tolerantes hacia la deuda son además las que más frecuentemente utilizan el endeudamiento como forma de acceso a los bienes y servicios, lo que concuerda con los hallazgos previos de Boddington y Kemp (1999), en cuanto a la correlación significativa entre la actitud hacia el endeudamiento y la tolerancia mayor hacia la deuda. Sin embargo, los resultados de estos estudios no proporcionan ninguna evidencia acerca de si el aumento de la deuda precedió al cambio de actitud, o si las dos medidas cambiaron al mismo tiempo. No obstante, independientemente de la relación causal entre la actitud hacia el endeudamiento y el nivel real de deuda de las personas, resulta evidente que existe una relación entre ambas variables, por lo cual se hace relevante el estudio de las actitudes hacia el endeudamiento.

Según Katona (1975), en cualquier economía moderna es casi imposible no tener una hipoteca sobre la casa, un crédito de consumo o una tarjeta de crédito, lo que varía considerablemente es cómo la gente acepta y utiliza estas formas de pago y hasta dónde las naturaliza como un evento cotidiano no cuestionado, lo que da origen al desarrollo de una cultura de endeudamiento, que tiene peligrosas 
implicancias sociales y económicas como el endeudamiento masivo (Denegri, 2007). Este ocasiona serios trastornos a los consumidores, afectando los ámbitos económico, financiero, familiar y social y caracterizándose por la pérdida de control personal de la situación económica financiera, el serio deterioro de las relaciones sociales construidas en torno al consumo y, por tanto, de las condiciones de integración y valoración social del consumidor endeudado (Castañeda, 2002).

En los últimos años, ha surgido un creciente interés por las prácticas financieras de los estudiantes universitarios, y específicamente por el aumento creciente del endeudamiento dado el riesgo que ello implica para su vida financiera y laboral después de su graduación. Numerosas investigaciones han examinado el uso de tarjetas de crédito como práctica financiera normal de los estudiantes universitarios, encontrando que esta es una práctica crecientemente independiente del nivel socioeconómico familiar (Barron \& Staten, 2004; Baum \& O’Malley, 2003; Chen \& Volpe, 2002; Davies \& Lea, 1995; Denegri, Martínez \& Etchebarne, 2007; Hayhoe, 2002; Hayhoe, Leach, Allen \& Edwards, 2005; Hayhoe, Leach \& Turner, 1999; Hayhoe, Leach, Turner, Bruin \& Lawrence, 2000; Jamba-Joyner, HowardHamilton \& Mamarchew, 2000; Lawrence et al., 2003; Lyons, 2003, 2004).

Otra serie de estudios realizados en poblaciones universitarias muestran la influencia potencial que tienen las actitudes hacia el crédito y las expectativas de futuro laboral, en el uso real de instrumentos de endeudamiento como las tarjetas de crédito (Baum \& O'Malley, 2003; Chien \& DeVaney, 2001; Davies \& Lea, 1995; Durkin, 2000; Hayhoe, 2002; Hayhoe et al., 1999; Norvilitis \& Santa Maria, 2002; Norvilitis, Szablicki \& Wilson, 2003; Xiao, Noring \& Anderson, 1995). Esto implica que para los estudiantes universitarios el endeudamiento actual puede ser considerado como una situación transitoria que les permite sustentar su actual estilo de vida, pero que sería superado una vez que aumenten sus ingresos al egresar de la universidad (Chien \& DeVaney, 2001; Lyons, 2004). Las variables actitudes hacia el crédito, género y dinero son predictores significativos que permiten discrimi- nar el uso de tarjetas de crédito en los estudiantes. Específicamente, los estudiantes que manifiestan actitudes positivas hacia el crédito también son los que manejaban un mayor número de tarjetas y, además, los que efectivamente se encuentran más endeudados, en comparación con aquellos que tienen menos tarjetas y una actitud neutra o negativa hacia el crédito (Hayhoe et al., 2000; Sharpe, Finke \& Weagley, 2005; Simon \& Whelan, 2003; Warwick \& Mansfield 2000; Xiao et al., 1995).

En relación con el uso de instrumentos de crédito, se observa que el porcentaje de endeudamiento aumenta progresivamente con la edad, con patrones diferenciales según grupo etáreo, apreciándose que en los más jóvenes (15 a 19 años) coexisten fuentes formales de endeudamiento (comercios y tarjetas de crédito adicionales) e informales como los amigos y la familia (Denegri, Palavecinos, Ripoll \& Yáñez, 1999; Medina, Méndez, Pérez \& Quezada, 1999; Ortega, Rodríguez-Vargas, Denegri \& Gempp, 2005).

En Estados Unidos, la Nellie Mae Corp. (2005) señala que el $76 \%$ de los estudiantes usa tarjeta de crédito y que un $47 \%$ tiene más de una, con una deuda promedio por estudiante de U\$2.169. Más recientemente, el Instituto Nacional de la Juventud de Chile ([INJUV], 2009), encontró que la mitad de la población juvenil chilena (50.6\%) tiene deudas impagas o por pagar, en tanto que el $45 \%$ asegura no tener deudas. En este sentido, la proporción de jóvenes con deudas desciende, significativamente, en el caso de créditos para la educación (16.1\%) y créditos hipotecarios (3.5\%), siendo las mujeres de los niveles socioeconómicos medios (C2 y C3), de sectores urbanos y de nivel técnico, quienes presentan un mayor nivel de endeudamiento. Estos resultados son similares a lo encontrado por Kemp, Horwood y Fergusson (2006) en una muestra de 1.265 estudiantes universitarios de Nueva Zelanda, de los cuales existía un nivel de endeudamiento del $50 \%$ del total de la muestra.

A partir de los estudios previos, la presente investigación tuvo como objetivo general, analizar la validez factorial de la Escala de Actitudes hacia el Endeudamiento (Denegri et al., 1999; Medina et al., 1999) y determinar la probable existencia de 
tipologías de perfiles actitudinales hacia el endeudamiento en estudiantes universitarios chilenos.

\section{Metodología}

\section{Participantes}

Se utilizó un muestreo de tipo no probabilístico por cuotas independientes. La muestra estuvo compuesta por 984 estudiantes universitarios de la ciudad de Temuco, Chile, cuyas edades fluctuaban entre 18 y 25 años, pertenecientes a los estratos socioeconómicos ABC1 (alto) $30 \%, \mathrm{C} 2$ y C3 (medio) $40 \%$ y D y $\mathrm{E}$ (bajo) $30 \%$, aspecto medido a través de la Matriz de Clasificación Socioeconómica (ESOMAR), la cual combina las variables nivel de educación alcanzado por el principal sostenedor del hogar y la categoría ocupacional de este (Adimark GfK \& Thompson, 2000).

\section{Instrumento}

La Escala de Actitudes hacia el Endeudamiento fue desarrollada por Denegri et al. (1999) (véase Apéndice), consta de 11 ítems en formato tipo Likert con cuatro opciones de respuesta, muy de acuerdo, de acuerdo, en desacuerdo y muy en desacuerdo, puntuando 1, 2, 3 y 4, respectivamente. Para su construcción se siguió una estrategia factorial que dio como resultado dos factores ortogonales, denominados Actitud Austera frente al endeudamiento y Actitud Hedonista frente al endeudamiento. Mientras la primera incluye actitudes de cautela y reserva frente al endeudamiento, la segunda incluye actitudes proclives a contraer deudas sin evaluar las consecuencias. Un análisis posterior con el objeto de ajustarla a población universitaria (Medina et al., 1999) reveló una estructura oblicua definida por tres factores que logran explicar el $40.3 \%$ de la varianza. Los dos primeros factores se corresponden bastante bien con el factor Austeridad reportado por Denegri et al. (1999) y parecieron constituir subcomponentes de este factor. A partir de los ítems salientes de cada factor, estos fueron denominados Temor al Endeudamiento y Precaución ante el Endeudamiento. El tercer factor, relativamente independiente de los anteriores, estaba compuesto por los mismos ítems que el factor de Hedonismo, encontrado por Denegri y su equipo.

Las propiedades psicométricas generales fueron evaluadas por Araneda, Brahm, García y Noriega (2005), encontrando que los índices de confiabilidad por consistencia interna, para cada uno de los factores de la escala, son adecuados. Los distintos índices de ajuste obtenidos confirmaron que la escala muestra una estructura bifactorial con dos dimensiones independientes $(\mathrm{GFI}=0.98$, RMSEA $=0.06$, otros índices de ajuste sobre 0.9).

TABLA 1

Varianza total explicada posteriormente a la eliminación de ítems a partir del análisis factorial exploratorio

\begin{tabular}{|c|c|c|c|c|c|c|c|c|c|}
\hline \multirow{2}{*}{ Factor } & \multicolumn{3}{|c|}{ Autovalores iniciales } & \multicolumn{3}{|c|}{$\begin{array}{l}\text { Sumas de las saturaciones de la } \\
\text { extracción }\end{array}$} & \multicolumn{3}{|c|}{$\begin{array}{l}\text { Suma de las saturaciones de la } \\
\text { rotación al cuadrado }\end{array}$} \\
\hline & Total & $\begin{array}{c}\% \text { de la } \\
\text { varianza }\end{array}$ & $\%$ acumulado & Total & $\begin{array}{c}\% \text { de la } \\
\text { varianza }\end{array}$ & $\%$ acumulado & Total & $\begin{array}{c}\% \text { de la } \\
\text { varianza }\end{array}$ & $\%$ acumulado \\
\hline 1 & 3.121 & 39.014 & 39.014 & 2.648 & 33.545 & 33.545 & 2.651 & 33.141 & 33.141 \\
\hline 2 & 1.617 & 20.208 & 59.222 & 1.049 & 13.108 & 46.653 & 1.081 & 13.512 & 46.653 \\
\hline 3 & 0.8 & 9.997 & 69.219 & & & & & & \\
\hline 4 & 0.696 & 8.699 & 77.918 & & & & & & \\
\hline 5 & 0.567 & 7.086 & 85.004 & & & & & & \\
\hline 6 & 0.476 & 5.954 & 90.958 & & & & & & \\
\hline 7 & 0.428 & 5.349 & 96.307 & & & & & & \\
\hline 8 & 0.295 & 3.693 & 100 & & & & & & \\
\hline
\end{tabular}

Nota. Método de extracción: mínimos cuadrados generalizados.

Fuente: elaboración propia. 


\section{Procedimiento}

Los administradores se acercaron individualmente a cada participante, para solicitar su colaboración voluntaria para responder la escala. Se les explicó el propósito general y se les entregó un formulario de consentimiento informado, en el cual se explican con mayor detalle los alcances de la investigación, posteriormente se entregó el material, clarificando que debían responderlo en forma individual. Finalmente, se fijó un plazo para la devolución del material. Una vez que el participante entregaba las escalas contestadas, se les agradecía su participación en el estudio.

\section{Resultados}

Previamente a la realización de los análisis, los datos fueron organizados en una matriz de sujetos por variables, en la que se invirtió la puntuación de los ítems, puesto que en ambos casos las escalas presentaban opciones de respuesta en sentido inverso, es decir, un formato de respuesta de 4 puntos, muy de acuerdo, de acuerdo, en desacuerdo y muy en desacuerdo, puntuando 1, 2, 3 y 4, respectivamente. Para facilitar la corrección, y de esta forma que una mayor puntuación refleje mayor nivel de actitud, se invirtieron los puntajes de respuesta, es decir, muy de acuerdo, de acuerdo, en desacuerdo y muy en desacuerdo, fueron puntuados 4, 3, 2 y 1 , respectivamente.

A fin de analizar la validez factorial de la escala se efectuaron análisis factoriales exploratorios y confirmatorios. De esta forma, a partir de la consideración simultánea de las comunalidades de los ítems y sus cargas factoriales en cada dimensión, se eliminaron sucesivamente los ítems 3,5 y 10. Asíla escala quedó compuesta por 8 ítems, de los cuales los ítems 4, 6, 7, 8, 9 cargan significativamente en el primer factor definido como Austeridad $(0.69 ; 0.7$; $0.54 ; 0.8$ y 0.86 , respectivamente), mientras que los ítems 1,2 y 11 saturan en el segundo factor denominado Hedonismo $(0.69 ; 0.64$ y 0.38 , respectivamente). Posteriormente a la eliminación de los ítems, el porcentaje de la varianza explicada por el Factor 1, Austeridad, corresponde a $33.14 \%$, mientras que el Factor 2, Hedonismo, explica el $13.51 \%$ de la varianza (Tabla 1 ), ratificando que la escala muestra una estructura bifactorial con dos dimensiones independientes $(\mathrm{GFI}=0.98$, $\mathrm{RMSEA}=0.06$, otros índices de ajuste sobre 0.90).

Por último, se realizó un análisis de clúster de doble conglomeración. En un inicio, se realizó un análisis de conglomerados jerárquico, mediante el cual se obtuvieron los centroides iniciales para los 4 grupos previamente establecidos (Tabla 2).

TABLA 2

Centroides iniciales de los conglomerados obtenidos a partir del análisis de clúster jerárquico

\begin{tabular}{lrrrc}
\hline & \multicolumn{4}{c}{ Conglomerado } \\
\cline { 2 - 5 } & 1 & 2 & 3 & 4 \\
\hline $\begin{array}{l}\text { Puntúa: } \\
\text { Austeridad }\end{array}$ & -3.78 & -0.53 & 0.773 & 0.773 \\
$\begin{array}{l}\text { Puntúa: } \\
\text { Hedonismo }\end{array}$ & -0.197 & 0.341 & -0.197 & 0.879 \\
\hline
\end{tabular}

Fuente: elaboración propia.

Posteriormente, se realizó un análisis de clúster aglomerativo no jerárquico de k-medias, con el objeto de refinar la clasificación obtenida a través del método jerárquico, para lo cual nuevamente se definió una solución inicial de 4 clústeres. Para este análisis, se utilizaron las variables austeridad y hedonismo, obtenidas a través del análisis factorial. Antes de realizar el análisis, se transformaron los puntajes factoriales a puntaciones $Z(M=0$, $D E=1)$, con el fin de eliminar el sesgo introducido por las diferencias de las mediciones de las puntuaciones obtenidas por los sujetos de la muestra. Para determinar la similitud respecto a los puntajes obtenidos para los factores Austeridad y Hedonismo, se utilizó la distancia euclídea al cuadrado. Las coordenadas de los centroides finales de los conglomerados (Figura 1), cuya abscisa corresponde al puntaje estandarizado de cada uno de los sujetos para el factor Austeridad y cuya ordenada corresponde al puntaje estandarizado para el factor Hedonismo, corresponden a: clúster 1 , difuso $(-3.62 ;-0.17)$; clúster 2 , hedonista $(-0.70 ; 0.48)$; clúster 3 , austero $(0.42 ;-0.88)$ y clúster 4 , ambivalente $(0.45 ; 0.88)$. 
Perfiles actitudinales

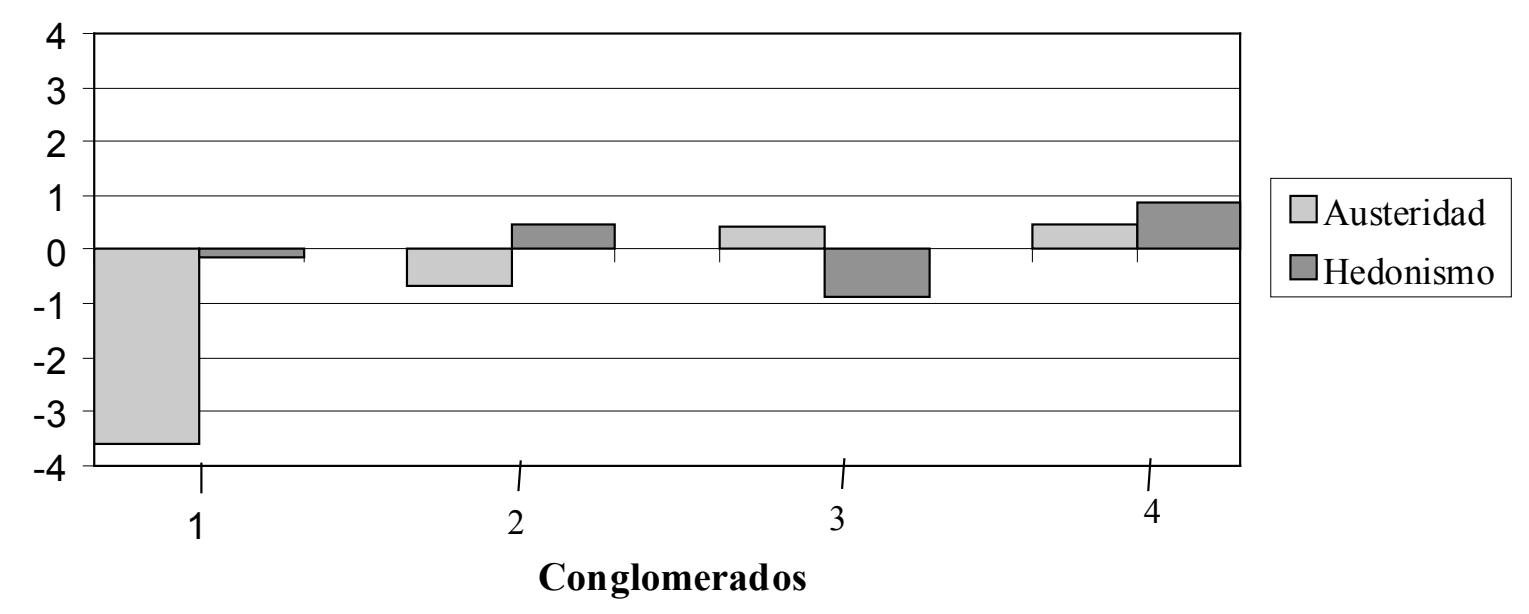

Figura 1. Representación de los perfiles actitudinales.

Fuente: elaboración propia.

De acuerdo a lo anterior, el clúster 1, difuso, se caracteriza por la presencia de puntajes muy por debajo de la media en el factor Austeridad y ligeramente bajos en el factor Hedonismo. En el clúster 2, hedonista, los sujetos presentan un puntaje ligeramente bajo la media en el factor Austeridad y ligeramente superior a la media en el factor Hedonismo. En el clúster 3, austero, los sujetos muestran un puntaje ligeramente superior a la media en el factor Austeridad y ligeramente inferior a la media en el factor Hedonismo y, por último, en el clúster 4, ambivalente, los sujetos presentan puntajes ligeramente sobre la media tanto en el factor Austeridad como Hedonismo.

A través de un análisis de varianza (ANOVA) con prueba post hoc (Tukey), se compararon las medias de los distintos clústeres en cada factor. Se encontró que para el Factor 1, Austeridad, el clúster 1 difuso y 2 hedonista, difieren de manera significativa; en tanto que entre los clúster 3 austero y 4 ambivalente no existen diferencias significativas. Para el Factor 2, Hedonismo, se encontraron diferencias significativas en todos los grupos (Tablas 3 y 4).

Desde los 4 clústeres definidos, se creó una nueva variable denominada Grupo, la cual muestra a qué clúster pertenece cada sujeto. Posteriormente, la variable Grupo fue sometida a nuevas pruebas estadísticas. Se calculó el porcentaje de sujetos incluidos en cada grupo, de esta forma el $4.5 \%$ de los sujetos de la muestra estaban incluidos en el clúster 1 (difuso), el $22.4 \%$ en el clúster 2 (hedonista), el $42.4 \%$ en el clúster 3 (austero) y el $30.7 \%$ en el clúster 4 (ambivalente).

Finalmente, se calculó la media de cada clúster a partir de los puntajes estandarizados para cada uno de los factores, a saber, Factor 1 (Austeridad) y Factor 2 (Hedonismo). De esta forma, las medias de los factores corresponden respectivamente a: clúster 1, difuso, $(\mathrm{M}=-3.62 ;-0.17)$; clúster 2, hedonista, $(\mathrm{M}=-0.69 ; 0.48)$; clúster 3 , austero, $(\mathrm{M}=0.42$; -0.88) y clúster 4 , ambivalente, $(M=0.45 ; 0.88)$.

\section{Discusión}

A partir de los análisis psicométricos realizados a la Escala de Actitudes hacia el Endeudamiento (Denegri et al., 1999), se encontró la presencia de dos factores independientes denominados Hedonismo y Austeridad, cuyas propiedades métricas son adecuadas. 
TABLA 3

Análisis de varianza post hoc de Tukey para el Factor 1, Austeridad

\begin{tabular}{|c|c|c|c|c|}
\hline \multirow{2}{*}{ Número inicial de casos } & \multirow{2}{*}{$\mathrm{N}$} & \multicolumn{3}{|c|}{ Subconjunto para alfa $=0,05$} \\
\hline & & 1 & 2 & 3 \\
\hline 1 & 53 & -3.62435 & & \\
\hline 2 & 263 & & -0.6936177 & \\
\hline 3 & 368 & & & 0.4233121 \\
\hline 4 & 300 & & & 0.4547294 \\
\hline Sig. & & 1.000 & 1.000 & 0.908 \\
\hline
\end{tabular}

Nota. Se muestran las medias para los grupos en los subconjuntos homogéneos.

${ }^{a}$ Usa el tamaño muestral de la media armónica $=145.688$.

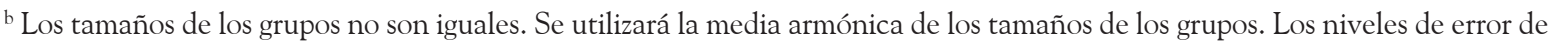
tipo I no están garantizados.

Fuente: elaboración propia.

TABLA 4

Análisis de varianza post hoc de Tukey para el Factor 2, Hedonismo

HSD de Tukey ${ }^{\mathrm{a}, \mathrm{b}}$

\begin{tabular}{|c|c|c|c|c|c|}
\hline \multirow{2}{*}{ Número inicial de casos } & \multirow{2}{*}{$N$} & \multicolumn{4}{|c|}{ Subconjunto para alfa $=0,05$} \\
\hline & & 1 & 2 & 3 & 4 \\
\hline 3 & 368 & -0.8757236 & & & \\
\hline 1 & 53 & & -1.660672 & & \\
\hline 2 & 263 & & & 0.4823373 & \\
\hline 4 & 300 & & & & 0.8834923 \\
\hline Sig. & & 1.000 & 1.000 & 1.000 & 1.000 \\
\hline
\end{tabular}

Nota. Se muestran las medias para los grupos en los subconjuntos homogéneos.

a Usa el tamaño muestral de la media armónica $=145.688$.

${ }^{\text {b }}$ Los tamaños de los grupos no son iguales. Se utilizará la media armónica de los tamaños de los grupos. Los niveles de error de tipo I no están garantizados.

Fuente: elaboración propia.

El análisis factorial confirmatorio ratifica el supuesto de que las actitudes hacia el endeudamiento evaluadas por la escala, arrojan la existencia de dos factores independientes denominados Hedonismo y Austeridad. De acuerdo a lo planteado por Kerlinger (1975 citado en Morales, 2000), es posible concluir que las actitudes significativas, como las actitudes hacia el endeudamiento, no parecen ser unidimensionales puesto que las creencias que determinan una posición favorable hacia el endeudamiento no son en esencia contrarias a las creencias que sustentan una posición desfavorable hacia el mismo. De esta forma las creencias acerca de las características asociadas a los objetos de consumo, junto a la importancia relativa asignada a estas, difieren en una misma persona, es decir, el sujeto puede estar más dispuesto a endeudarse al adquirir un producto asociado a ciertas características valoradas positivamente por él y en menor medida al adquirir otro producto cuyas características no son tan valoradas. Lo anterior parece estar vinculado al hecho de que los productos y servicios no son consumidos atendiendo exclusivamente a sus propiedades funcionales, sino también a los beneficios de carácter psicológico y social que reportan a sus usuarios (Ortega et al., 2005). 
A partir del análisis de ítems realizado, se encontró una tendencia de respuesta distinta en cada uno de los factores. Mientras el factor Hedonismo tiende a los puntajes medios (en desacuerdo y de acuerdo), el factor Austeridad muestra una propensión a los puntajes altos (de acuerdo y muy de acuerdo). Dados los altos niveles de endeudamiento, cabe preguntarse si la tendencia a los puntajes altos en el factor Austeridad está influenciada por la deseabilidad social, de esta forma, en los sujetos de la muestra podría existir una tendencia a atribuirse a sí mismos actitudes socialmente deseables y rechazar aquellas socialmente indeseables. Esto podría encontrarse vinculado a los valores más tradicionales que otorgan mayor relevancia a la austeridad como forma de vida y que corresponde a estereotipos culturales previos a la implantación del modelo neoliberal y a los rápidos cambios económicos (Denegri et al., 1999).

Finalmente, el análisis se orientó a establecer una tipología de perfiles actitudinales hacia el endeudamiento, partiendo del supuesto de la existencia de dos factores independientes medidos por la escala, a saber, Hedonismo y Austeridad. A través del análisis de conglomerados fue posible determinar cuatro perfiles de actitudes hacia el endeudamiento, los cuales se establecen a partir de una matriz que combina ambos factores. Esta matriz surge de la combinación de sujetos que exhiben, en distintos grados, alto o bajo nivel de Hedonismo y un alto o bajo nivel de Austeridad. De acuerdo a lo anterior, el clúster 1 (difuso) se caracteriza por la presencia de puntajes muy por debajo de la media en el factor Austeridad y ligeramente bajos en el factor Hedonismo. En el clúster 2 (hedonista), los sujetos presentan un puntaje ligeramente bajo la media en el factor Austeridad y ligeramente superior a la media en el factor Hedonismo. En el clúster 3 (austero) los sujetos muestran un puntaje ligeramente superior a la media en el factor Austeridad y ligeramente inferior en el factor Hedonismo y, por último, en el clúster 4 (ambivalente) los sujetos presentan puntajes ligeramente sobre la media tanto en el factor Austeridad como Hedonismo. De acuerdo a lo anterior, es posible definir conceptualmente los perfiles actitudinales mencionados de la siguiente manera:

El perfil hedonista corresponde al consumidor en el que predominan actitudes positivas hacia el uso del crédito y el endeudamiento (Denegri et al., 1999).

El perfil austero se caracteriza por presentar un estilo actitudinal en el que se enfrenta con cautela al endeudamiento y se orienta hacia un manejo más tradicional de los recursos financieros, es decir, basado en el ahorro y la evitación del crédito (Denegri et al., 1999).

El perfil ambivalente presentaría una coexistencia de actitudes que valoran el ahorro y el uso mesurado de los recursos, y actitudes asociadas a la concepción del uso del crédito como una vía de acceso rápido a las necesidades de compra.

El perfil difuso estaría conformado por sujetos cuyo perfil actitudinal aún no se encuentra definido, por lo que podría tratarse de sujetos cuyas actitudes se encuentran en proceso de transición y que, por lo tanto, pueden evolucionar indistintamente a cualquiera de los tres perfiles antes mencionados.

A partir de los análisis realizados se encontró que los promedios de cada perfil difieren entre sí, no obstante, los estilos no definen tendencias extremas. La mayoría de los sujetos exhiben un perfil predominantemente austero (42.4\%) y en segundo lugar Ambivalente (30.7\%). El mayor porcentaje de sujetos pertenecientes al perfil austero puede estar relacionado con la procedencia de los individuos de la muestra, quiénes residen desde su infancia en la ciudad de Temuco, una ciudad del sur de Chile en una región de fuerte tradición agrícola y de latifundio, la cual si bien ha alcanzado un mayor desarrollo económico y financiero, coexisten en ella fuertes tendencias culturales tradicionales ligadas a la valoración de la austeridad como forma de vida, la importancia del trabajo y el valor del esfuerzo y el sacrificio, así como un cierto rechazo a la ostentación y el lujo. Estos valores y estereotipos culturales previos a la implementación del modelo neoliberal, parecen entrar en conflicto con los valores imperantes en las modernas sociedades de consumo, donde el crédito efectivamente se ha transformado en una característica consustancial 
a los patrones de marketing y comercialización de bienes y servicios (Denegri et al., 1999), resultado concordante con lo encontrado en otros estudios en poblaciones en proceso de cambio cultural (AbdulMuhmin, 2008).

Si consideramos además que la muestra está constituida por jóvenes, el alto porcentaje de individuos pertenecientes al clúster 3 (ambivalente), puede explicarse por la presencia del cambio cultural desde patrones más austeros y tradicionales a patrones más hedonistas y consumistas. Así, los individuos si bien fueron socializados tempranamente con los valores tradicionales, se encuentran en un proceso de transición y de apropiación de los nuevos valores asociados a la sociedad de consumo actual, los cuales se orientan a la búsqueda de la satisfacción inmediata de necesidades de consumo y a una hipervaloración de este como soporte de la identidad e integración social, lo que lleva a los jóvenes a una valoración y justificación del uso del crédito como una práctica cultural validada (Denegri et al., 1999; Hayhoe et al., 2000; Winerman, 2004; Xiao et al., 1995).

De acuerdo a lo anterior, es posible inferir que los sujetos pertenecientes a los perfiles actitudinales ambivalente y difuso, serían los más susceptibles de ser afectados por los factores situacionales, a la hora de tomar una decisión de compra. Esta susceptibilidad se entiende en el primer grupo de sujetos, por la coexistencia de actitudes proclives y contrarias a la deuda y en el segundo grupo, por el hecho de no tener una actitud clara que regule su conducta de compra y endeudamiento, por lo tanto, en ambos perfiles actitudinales serán los factores contextuales los que finalmente determinarán la conducta de deuda del individuo (Barron \& Staten, 2004; Baum \& O’Malley, 2003; Chien \& DeVaney, 2001; Davies \& Lea, 1995; Durkin, 2000; Hayhoe, 2002; Hayhoe et al., 1999), lo que también aumenta su potencial de riesgo frente a la presión del mercado (Jamba-Joyner et al., 2000; Lawrence et al., 2003, Lyons, 2003, 2004) y atenta contra la calidad de su futura vida financiera, posterior a la inserción laboral después de su graduación.

Finalmente, en un sentido socio-cultural y económico-político más amplio, este trabajo se vincula y nos permite la reflexión sobre una problemática común en el espacio latinoamericano, al abordar el impacto que ha tenido en nuestras poblaciones la instalación de un modelo económico y de mercado que presiona a los individuos a construir sus identidades en torno al consumo y donde los valores se orientan al corto plazo, la satisfacción inmediata y el abandono de la valoración del ahorro y de la previsibilidad, para dar lugar a la naturalización del endeudamiento y el gasto continuo (Bauman, 2007).

A la vez, releva la importancia de diseñar e implementar políticas públicas de educación que se orienten a la alfabetización financiera y al desarrollo de una ciudadanía económica reflexiva, temática ausente de los curriculums educativos en nuestros contextos, "considerando el problema estratégico que tiene la apropiación del conocimiento en la valorización y reproducción de la vida social y humana y los sistemas de dominación" (Sierra, 2006, pp. 275 y 276). Tal como lo menciona García Canclini (1995), el consumo dejó de ser una actividad humana orientada a la satisfacción de necesidades convirtiéndose en el gran articulador de la construcción de una ciudadanía mediada por él. En este nuevo espacio, el individuo percibe que muchas de las preguntas que orientaban el ejercicio ciudadano como la pertenencia social, los derechos, la delegación del poder y la representatividad y necesidad de participar y estar informados, pueden satisfacerse en el espacio del consumo privado y de los medios de comunicación, más que en las reglas abstractas de la democracia o de la participación ciudadana. En este escenario, incluso la participación política o la toma de decisiones en este ámbito, se ve mediatizada y modelada por las estrategias publicitarias donde el elector se siente convocado como consumidor aún cuando se le solicite como ciudadano.

Desde esta perspectiva, se podría hipotetizar que la emergencia de los perfiles ambivalente y difuso, que se revelan en esta investigación, podría ser un fenómeno que refleja no solo posturas ante el endeudamiento, sino probablemente se vincula a estilos actitudinales más amplios en otros temas de la vida social, donde una parte importante de la población toma sus decisiones influenciada por variables del contexto del momento o por el uso de 
heurísticos (Kahneman, 2003), más que por el análisis racional de las diversas alternativas presentes.

Es así como la investigación orientada a la comprensión de la cultura latinoamericana actual, implica el reconocimiento de la centralidad que tienen las problemáticas asociadas al consumo en la construcción de las identidades de nuestras sociedades. De hecho, al pensar lo procomún, uno de los tópicos articuladores es el que se ha convenido en llamar capitalismo cognitivo (Sierra, 2011), señalando el impacto del modelo económico no solo en la toma de decisiones de compra, sino también en la construcción de mentalidades e imaginarios sociales que orientan y modelizan la conducta personal y colectiva (Vázquez, 2005).

\section{Referencias}

Abdul-Muhmin, A. G. (2008). Consumer attitudes towards debt in an Islamic country: Managing a conflict between religious tradition and modernity? International Journal of Consumer Studies, 32( 3), 194-203.

Adimark GfK \& Thompson, W. (2000). El nivel socioeconómico. Manual de aplicación. Extraído el 12 de septiembre de 2004, de www.adimark.cl

Amar, J., Abello, R., Denegri, M. \& Llanos, M. (2007). Pensamiento Económico en jóvenes universitarios. Revista Latinoamericana de Psicología, 39(2), 363-373.

Araneda, P., Brahm, C., García, C. \& Noriega, A. (2005). Tipologías de Perfiles Actitudinales hacia el Endeudamiento. Tesis de Licenciatura en Psicología, Universidad de La Frontera, Temuco, Chile.

Avard, S., Manton, E., English, D. \& Walker, J. (2005). The financial knowledge of college freshmen. College Student Journal, 39(2), 321-339.

Barron, J. M. \& Staten, M. E. (2004). Usage of credit cards received through collage student-marketing programs. Journal of Student Financial Aid, 34(3), 7-26.

Baum, S. \& O'Malley, M. (2003). College of credit: How borrowers perceive their education debt. Journal of Student Financial Aid, 33(3), 7-19.

Bauman, Z. (2007). Vida de consumo. Buenos Aires: Fondo de Cultura Económica.
Boddington, L. \& Kemp, S. (1999). Student debt, attitudes towards debt, impulsive buying, and financial management. New Zealand Journal of Psychology, 28(2), 89-93.

Castañeda, P. (2002). El endeudamiento como problemática social emergente: el caso de los consumidores de Valparaíso Metropolitano. Ponencia presentada en el Congreso Internacional de Políticas Sociales, Universidad del Biobío, Concepción. Extraído el 15 de agosto de 2005, de http://www.ubiobio.cl/cps/ ponencia/doc/p4.1.htm

Chen, H. \& Volpe, R. P. (2002). Gender differences in personal financial literacy among college students. Financial Services Review, 11(3), 289-307.

Chien, Y. \& DeVaney, S. A. (2001). The effects of credit attitude and socioeconomic factors on credit card and installment debt. The Journal of Consumer Affairs, 35, 179-162.

Davies, E. \& Lea, S. E. (1995). Student attitudes to student debt. Journal of Economic Psychology, 16, 663-679.

Denegri, M. (2007). Introducción a la psicología económica. Colombia: PSICOM Editores. ISBN: 978958-98153-6-6

Denegri, M., Martínez, G. \& Etchebarne, S. (2007). La comprensión del funcionamiento bancario en adolescentes chilenos: un estudio de psicología económica. Interdisciplinaria, 24(2), 137-160.

Denegri, M., Palavecinos, M., Gempp, F. R. \& Caprile, C. (2005). Socialização econômica em famílias chilenas de classe média: educando cidadãos ou consumidores? Psicología e Sociedade, 17(2), 88 -98.

Denegri, M., Palavecinos, M., Ripoll, M. \& Yáñez, V. (1999). Caracterización psicológica del consumidor de la IX región. En M. Denegri, F. Fernández, R. Iturra, M. Palavecinos \& M. Ripoll (Eds.), Consumir para vivir y no vivir para consumir (pp. 7-31). Temuco, Chile: Ediciones Universidad de La Frontera.

Descouvières, C. (1998). Psicología económica. Santiago, Chile: Editorial Universitaria.

Durkin, T. A. (2000). Credit cards: Use and consumer attitudes, 1970-2000. Federal Reserve Bulletin, 623634. 
García Canclini, N. (1995). Consumidores y ciudadanos. Conflictos multiculturales de la globalización. México: Grijalbo.

Godwin, D. D. (1997). Dynamics of households' income, debt, and attitudes toward credit, 1983-1989. Journal of Consumer Affairs, 31(2), 303-325.

Hayhoe, C. R. (2002). Comparison of affective credit attitude scores and credit use of college students at two points in time. Journal of Family and Consumer Science, 94(1), 71-77.

Hayhoe, C. R., Leach, L., Allen, M. W. \& Edwards, R. (2005). Credit cards held by college students. Financial Counseling and Planning, 16(1), 1-10.

Hayhoe, C. R., Leach, L. \& Turner, P. R. (1999). Discriminating the number of credit cards held by college students using credit and money attitudes. Journal of Economic Psychology, 20, 643-656.

Hayhoe, C. R., Leach, L. J., Turner, P. R., Bruin, M. J. \& Lawrence, F. C. (2000). Differences in spending habits and credit use of college students. Journal of Consumer Affairs, 34(1), 113-133.

Instituto Nacional de la Juventud. (2009). Sexta encuesta nacional de juventud: capítulo endeudamiento. Resultados preliminares. Santiago, Chile: Autor.

Jamba-Joyner, L. A., Howard-Hamilton, M. \& Mamarchew, H. (2000). College students and credit cards: Cause for concern. NASFAA Journal of Student Financial Aid, 30, 17-25.

Kahneman, D. (2003). Mapas de racionalidad limitada: psicología para una economía conductual. Revista Asturiana de Economía, 28, 181-225.

Katona, G. (1975). Psicología de la economía. Buenos Aires: Editorial El Ateneo.

Kemp, S., Horwood, J. \& Fergusson, D. (2006). Student loan debt in a New Zealand cohort study. New Zealand Journal of Educational Studies, 41(2), 273-291.

Kim, H. \& DeVaney, S. A. (2001). The determinants of outstanding balances among credit card revolvers. Financial Counseling and Planning, 12(1), 67-78.

Lawrence, F. C., Christofferson, S. Nester, B., Moser, J. A., Tucker, J. A. \& Lyons, A. C. (2003). Credit card usage of college students: Evidence from Louisiana State University. Research Information Sheet Number, 107, 1-28.
Lea, S., Webley, P. \& Levine, M. (1993). The economic psychology of consumer debt. Journal of Economic Psychology, 14, 85-119.

Lea, S., Webley, P. \& Walker, C. M. (1995). Psychological factor in consumer debt: Money management, economic socialization, and credit use. Journal of Economic Psychology, 16, 681-701.

Livingstone, S. \& Lunt, P. (1992). Predicting personal debt and debt repayment: Psychological, social and economic behavior. Journal of Economic Psychology, $13,111-134$.

Luna, R. (1998). Dinero, trabajo y consumo. Valencia, España: Promolibro.

Lyons, A. C. (2003). The credit usage and financial education needs of Midwest college students (Report released on December). Urbana-Champaign, IL: University of Illinois.

Lyons, A. C. (2004). A profile of financially at-risk college students. The Journal of Consumer Affairs, 38(1), 56-80.

Medina, M., Méndez, G., Pérez, C. \& Quezada, J. (1999). Evaluación de conductas y actitudes hacia el endeudamiento en estudiantes de la Universidad de La Frontera. Tesis de título profesional y Licenciatura en Psicología, Universidad de La Frontera, Temuco, Chile.

Morales, P. (2000). Medición de actitudes y educación: construcción de escalas y problemas metodológicos. Madrid: Universidad Pontificia Comillas.

Nellie Mae Corporation. (2005, mayo). Undergraduate students and credit cards in 2004: An analysis of usage rates and trends. Extraído el 25 de enero, 2007, de http://www.nelliemae.com/library/research_12.html

Norvilitis, J. M. \& Santa Maria, P. (2002). Credit card debt on college campuses: Causes, consequences, and solutions. College Student Journal, 36, 357-364.

Norvilitis, J. M., Szablicki, P. B. \& Wilson, S. D. (2003). Factors influencing levels of credit-card debt in college students. Journal of Applied Social Psychology, 33(5), 935-947.

Ortega, V., Rodríguez, J., Denegri, M. \& Gempp, R. (2005). Segmentación psicoeconómica: obtención y validación de perfiles para consumidores adultos jóvenes de nivel socioeconómico medio y alto en 
Colombia. Revista Internacional de Ciencias Sociales y Humanidades, 15(2), 117- 135.

Sharpe, D. L., Finke, M. S. \& Weagley, R. O. (2005, noviembre). Relationship between credit card management and likelihood that financial situation will make college completion difficult. Proceedings of the Annual Conference of the Association for Financial Counseling and Planning Education, Scottsdale, AZ, United States.

Sierra, F. (2006). Políticas de comunicación y educación. Crítica y desarrollo de la sociedad del conocimiento. Barcelona: Gedisa.

Sierra, F. (2011). Cultura latina y sociedad de la información. Pensar lo procomún. En C. Del Valle, F. Moreno \& F. Sierra (Eds.), Cultura latina y revolución digital. Matrices para pensar el espacio iberoamericano de comunicación (pp. 69-93). Barcelona: Gedisa.

Simon, R. \& Whelan, C. (2003, septiembre 3). The new credo on campus: "Just charge it" - credit card companies target students before freshman year; should your kid get one? Wall Street Journal, p. D1.
Vázquez, F. (2005). "Empresarios de nosotros mismos". Biopolítica, mercado y soberanía en la gubernamentalidad neoliberal. En J. Ugarte (Comp.), La administración de la vida. Estudios biopolíticos (pp. 73-103). Barcelona: Anthropos.

Wärneryd, K. -E. (1999). The psychology of saving: A study on economic psychology. Cheldenham, England: Rdward Elgar Publishing.

Warwick, J. \& Mansfield, P. (2000). Credit card consumers: College students' knowledge and attitude. Journal of Consumer Marketing, 17(7), 617-626.

Watson, J. (2003). The relationship of materialism to spending tendencies, saving, and debt. Journal of Economic Psychology, 24, 723-739.

Winerman, L. (2004, junio). Maxed out: Why do some consumers and others steer clear? Monitor on Psychology, 35(6), 62.

Xiao, J. J., Noring, F. E. \& Anderson, J. G. (1995). College students' attitudes towards credit cards. Journal of Consumer Studies and Home Economics, 19, 155-174.

Zuckerman, G. (2000, julio 5). Borrowing levels reach a record, sparking debate. The Wall Street Journal, pp. C1-18. 


\section{Apéndice}

Escala original de Actitudes hacia el Endeudamiento (Denegri et al., 1999)

A continuación señale el número que mejor refleja su grado de acuerdo o desacuerdo con esta afirmación según el cuadro que se indica a continuación:

\begin{tabular}{lll}
\hline Muy de acuerdo & $=$ & 1 \\
\hline De acuerdo & $=$ & 2 \\
\hline En desacuerdo & $=$ & 3 \\
\hline Muy en desacuerdo & $=$ & 4 \\
\hline
\end{tabular}

\begin{tabular}{ll}
\hline Afirmaciones & Grado de Acuerdo \\
\hline 1. Usar el crédito permite tener una mejor calidad de vida. & \\
\hline 2. Es una buena idea comprar algo ahora y pagarlo después. & \\
\hline 3. El uso del crédito puede ser muy peligroso. \\
\hline 4. Es preferible tratar de pagar siempre al contado. \\
\hline 5. El uso del crédito es una parte esencial del estilo de vida actual. \\
\hline 6. Es importante tratar de vivir de acuerdo al dinero que se tiene. \\
\hline 7. Si uno se lo propone, siempre puede ahorrar algo de dinero. \\
\hline 8. Es importante pagar las deudas lo antes posible. \\
\hline 9. Hay que ser muy cuidadoso en el gasto del dinero. \\
\hline 10. La facilidad de obtener tarjetas de crédito es una causa del endeudamiento de la gente. \\
\hline 11. Pedir un préstamo es a veces una muy buena idea.
\end{tabular}


